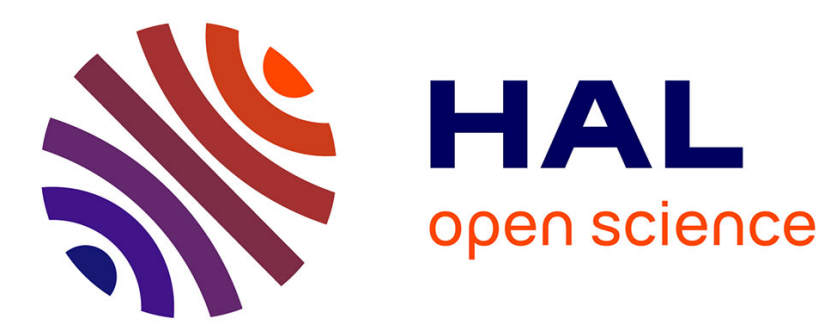

\title{
Computing methods of hypersingular integral applied to eddy-current testing
}

Philippe Beltrame, Noël Burais

\section{To cite this version:}

Philippe Beltrame, Noël Burais. Computing methods of hypersingular integral applied to eddy-current testing. IEEE Transactions on Magnetics, 2002, 38 (2 Part 1), pp.1269-1272. hal-00140342

\section{HAL Id: hal-00140342 \\ https://hal.science/hal-00140342}

Submitted on 6 Apr 2007

HAL is a multi-disciplinary open access archive for the deposit and dissemination of scientific research documents, whether they are published or not. The documents may come from teaching and research institutions in France or abroad, or from public or private research centers.
L'archive ouverte pluridisciplinaire HAL, est destinée au dépôt et à la diffusion de documents scientifiques de niveau recherche, publiés ou non, émanant des établissements d'enseignement et de recherche français ou étrangers, des laboratoires publics ou privés. 


\title{
Computing Methods of Hypersingular Integral Applied to Eddy-Current Testing
}

\author{
Ph. Beltrame and N. Burais
}

\begin{abstract}
The detection of thin-opening cracks is an important part of the eddy-current nondestructive testing (NDT). The integral formulation is well adapted for this modelization if the geometry of the tested piece is simple. However, some integrals involved in the computation contain strong singularities. The aim of this paper is to improve the classical numerical resolution using a general computing method of hypersingular integral.
\end{abstract}

Index Terms-Eddy-current, hypersingular integral, modelization, nondestructive testing.

\section{INTRODUCTION}

$\mathbf{E}$ DDY-CURRENT testing for the detection of cracks is widely used in the inspection of aeroplanes wings or of tubes in nuclear power plants. A driving coil generates a time-varying field inducing eddy-current in a conducting piece (Fig. 1). A flaw perturbs the eddy-current distribution and, hence, the coil impedance $Z$. Numerical methods to solve Maxwell's equations are required to evaluate this perturbation. The field computation is fully three-dimensional (3-D) and reveals important variations of the electromagnetic field near crack [1].

In order to tackle this problem, we consider the perturbation problem: the difference between the unperturbed problem and the full problem with crack. The flaw is the secondary current source. A boundary integral method is used, thus only the flaw domain has to be meshed. For simple geometries of the tested piece (without crack), the elementary solution (dyadic Green function) can be found out. An integral equation of the current source is obtained (volume integral method). A common approximation is that crack width is neglected and that eddy-current cannot flow through the flaw. So, the crack is equivalent to a dipole current surface, which is solution of a Fredholm's equation with a hypersingular kernel [2]. Only piecewise constant dipole densities are used [2], [3] because it is difficult to accurately evaluate the hypersingular integral.

This paper is devoted to the numerical resolution of this integral equation. In Section II, we give a simple picture of hypersingular integration and the main idea of regularization methods. Section III presents two regularized formulas of hypersingular integral for arbitrary shape functions, under existence requirements. In Section IV, constant, bilinear, and quadratic shape functions are used and their effectiveness is compared.

Manuscript received July 5, 2001.

The authors are with the Ecole Centrale Lyon-CEGELY. UMR C.N.R.S 5005 69131-Ecully Cedex, France (e-mail: beltrame@eea.ec-lyon.fr; burais@eea.ec-lyon.fr).

Publisher Item Identifier S 0018-9464(02)01225-6.

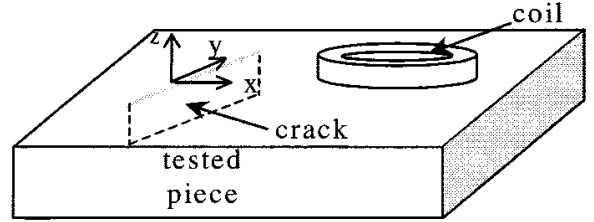

Fig. 1. Schematic configuration for the crack detection. Team Workshop Problem 15.

\section{FORMULATION}

The integral equation of the dipole density $p$ is in a slightly different form than in [2]

$$
\begin{aligned}
\forall \mathbf{r}_{0} \in S: J_{n}^{0}\left(\mathbf{r}_{0}\right)=\lim _{\mathbf{r}^{\prime} \rightarrow \mathbf{r}_{0}} \iint_{S} \Delta_{T} g_{0}\left(R_{T}\right) p(\mathbf{r}) d S_{r} \\
-k^{2} \iint_{S} g_{R}\left(R^{+}\right) p(\mathbf{r}) d S_{r}
\end{aligned}
$$

where $J_{n}^{0}$ is the incident current in the plate, $k$ is the propagation constant of the tested piece, $\mathbf{r}_{0}$ is the projection of $\mathbf{r}^{\prime}$ on the crack surface $S, R_{T}=\left|\mathrm{r}-\mathrm{r}^{\prime}\right|, R=\left|\mathrm{r}-\mathrm{r}_{0}\right|, R^{+}$is the distance between $\mathbf{r}_{0}$ and the image source $\left(R^{+}\right.$does not vanish except at the crack mouth), $\Delta_{T}=\partial^{2} / \partial y^{2}+\partial^{2} / \partial z^{2}$ is the tangential Laplacian, $g_{0}(R)=\exp (j k R) / 4 \pi R$ is the scalar free-space Green function, and, finally, $g_{R}$ contains reflection terms of Green function.

The second integral of (1) is regular, however, the first one has a hypersingular kernel $\left(1 / R^{3}\right.$ term). Let us denote $I_{S}$ the limit on the observation point $\mathbf{r}^{\prime}$ of the integral of the source term. A fundamental step is the transformation of this limit into an integral of the finite part of Hadamard $(F P)$ sense

$$
I_{S}=F P \iint_{S} \Delta_{T} g_{0}(R) p(\mathbf{r}) d S_{r} .
$$

However, in generic case, a jump term [4] must be added to FP. In our case, it vanishes (see the Appendix). The calculation of such integrals is not easy and a Gauss quadratic scheme leads to important numerical errors. In order to understand the specificity of this integration, we give a simple picture of the integration in the electrostatic case.

\section{Simple Picture of InTEGRATION IN SOURCE REgion}

The presence of singularity in the integral ensues from the source kind. In the present case, the source is a surface current dipole, i.e., two elementary currents in opposite directions. Let us consider the electrostatic case. An elementary current is equivalent to an electrostatic charges dipole $p_{1}$ with a pair of 


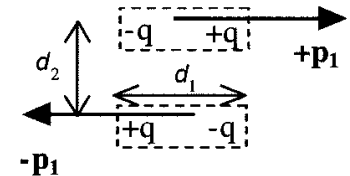

Fig. 2. Punctual electrostatic quadrupole.

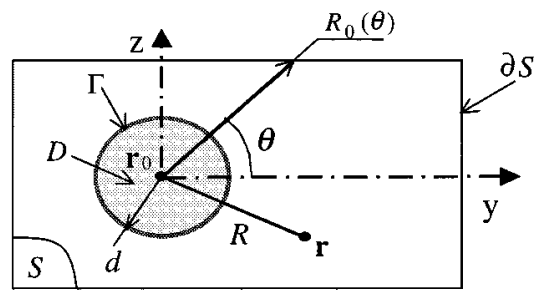

Fig. 3. Polar coordinates, and the exclusion disk $D$ for the $F P$ integration.

opposite charges $q$ at the ends of the current element. Then, the current dipole is equivalent to a quadrupole (Fig. 2). The quadrupole moment is given by: $p_{2}=d_{2} p_{1}$, where $p_{1}$ is the electrostatic dipole moment: $p_{1}=d_{1} q$. Each charge and dipole density diverges separately when $d_{1}$ and $d_{2}$ tend to zero, however, they vanish in the whole quadrupole. Indeed, the singular surface density associated to the punctual quadrupole is [5]: $\rho=-(1 / 2 \pi) d_{2} \Delta_{T} \delta$, where $\delta$ is the Dirac distribution. It can be proved: $\iint_{S} \rho d S=0$, and $\iint_{S} \rho R d S=0$, i.e., the total charges and dipoles moments vanish.

In the finite part integration, charges and dipoles terms must be regrouped together. After integration, they may vanish.

\section{REgUlarization MEthods}

We assume that the dipole density $p$ verify the regularity requirement described in [6]. This regularity property is required by the nature of hypersingularity, no matter what method used. It is practical to introduce polar coordinates $(R, \theta)$ center at $\mathbf{r}_{0}$ (Fig. 3). Two regularization methods are proposed.

\section{A. Regularization of Second-Order}

This method is based on the Guiggiani algorithm [6]. We used the expression (12) proved in the Appendix

$$
I_{S}=\lim _{d \rightarrow 0}\left(\iint_{S-D} \Delta_{T} g_{0}(R) p(\mathbf{r}) d S_{r}-\frac{2 \pi}{d} p\left(\mathbf{r}_{0}\right)\right) .
$$

1) Isolating the Singularities: Let us write the series expansion with respect to $R$ of the integrand of (3)

$$
\Delta_{T} g_{0}(R) p(\mathrm{r})=\frac{p\left(\mathrm{r}_{0}\right)}{R^{3}}+\frac{f(\theta)}{R^{2}}+\left(\frac{p_{2}(\mathbf{r})}{R^{2-\alpha}}+\frac{1}{R} g_{2}(R) p(\mathbf{r})\right)
$$

where

$$
f(\theta)=\left.\frac{\partial p}{\partial y}\right|_{\mathbf{r}=\mathbf{r}_{0}} \cos \theta+\left.\frac{\partial p}{\partial z}\right|_{\mathbf{r}=\mathbf{r}_{0}} \sin \theta
$$

and $g_{2}, p_{2}$ are bounded functions on $S$ and depend on $g$ and $p$, respectively. The terms in parentheses are weakly singular, thus we can take the limit $d=0$. We study the integrals $I_{1}$ and $I_{2}$ where the integrands are first and second term of (4), respectively.

2) Regrouping Terms Together:

$$
\begin{aligned}
I_{1} & =\iint_{S} \frac{f(\theta)}{R^{2}} d S_{r} \\
& =\ln (d) \int_{0}^{2 \pi} f(\theta) d \theta-\int_{0}^{2 \pi} f(\theta) \ln \left(R_{0}(\theta)\right) d \theta .
\end{aligned}
$$

We have $\int_{0}^{2 \pi} f(\theta) d \theta=0$, so the divergent dipole term vanishes

$$
I_{2}=\iint_{S-D} \frac{p\left(\mathbf{r}_{0}\right)}{R^{3}} d S_{r}=\frac{2 \pi}{d} p\left(\mathrm{r}_{0}\right)-p\left(\mathbf{r}_{0}\right) \int_{0}^{2 \pi} \frac{d \theta}{R_{0}(\theta)} .
$$

The first one diverges (charges term), however, vanishes with the divergent term of (3). The second is called a free-term.

Finally, the integral $I_{S}$ can be expressed only using regular or weakly singular integrals

$$
\begin{aligned}
I_{S}=-p\left(\mathbf{r}_{0}\right) & \int_{0}^{2 \pi} \frac{d \theta}{R_{0}(\theta)}+\int_{0}^{2 \pi} f(\theta) \ln \left(R_{0}(\theta)\right) d \theta \\
& +\iint_{S}\left(\frac{p^{(2)}(\mathbf{r})}{R^{2-\alpha}}+\frac{1}{R} g^{(2)}(R) p(\mathbf{r})\right) d S_{r} .
\end{aligned}
$$

\section{B. Integration by Parts and First-Order Regularization}

Integration by parts of a slightly modified form of (3) decreases the singularity

$$
I_{S}=-\lim _{d \rightarrow 0}\left(\iint_{S-D} \frac{\partial g_{0}}{\partial R} \frac{\partial p}{\partial R} d S_{r}\right)+\oint_{\partial S} \frac{\partial G}{\partial n} p(\mathbf{r}) d s .
$$

This integral is interpreted as the Cauchy principal value (CPV). The same method as previous adapted to CPV gives the regularized form

$$
\begin{aligned}
I_{S}=\int_{0}^{2 \pi} f(\theta) & \ln \left(R_{0}(\theta)\right) d \theta+\oint_{\partial S} \frac{\partial G}{\partial n} p(\mathbf{r}) d s \\
& +\iint_{S}\left(\frac{p^{(2)}(\mathbf{r})}{R^{2-\alpha}}+\frac{1}{R} g^{(2)}(R) p(\mathbf{r})\right) d S_{r} .
\end{aligned}
$$

The curvilinear integral on the boundary $\partial S$ is regular because the point $\mathrm{r}_{0}$ is not on $\partial S$.

\section{Validation}

In order to compute weakly singular integrals in (7) and (9), polar transform $(R, \theta)$ is applied. Then, they are computed by a Gaussian quadrature scheme.

The formulas (7) and (9) are validated for the particular case: $g_{0}(R)=1 / R, p(y, z)=y^{2}$, and $S$ is a circle of unit radius. Analytically, $I_{S}=\pi$ and using (7) and (9) we find $I_{S} \cong 3.1416$.

\section{NUMERICAL SCHEMES}

Equation (1) is solved by a collocation method. The variational formulation is longer because it requires a double surface integration. The surface crack is discretized by a regular mesh with rectangular elements. This regular mesh allows to decrease the size of elementary matrix [2]. Let us introduce the global numbering $n$ of nodes and the local numberings $(e, j)$ where 


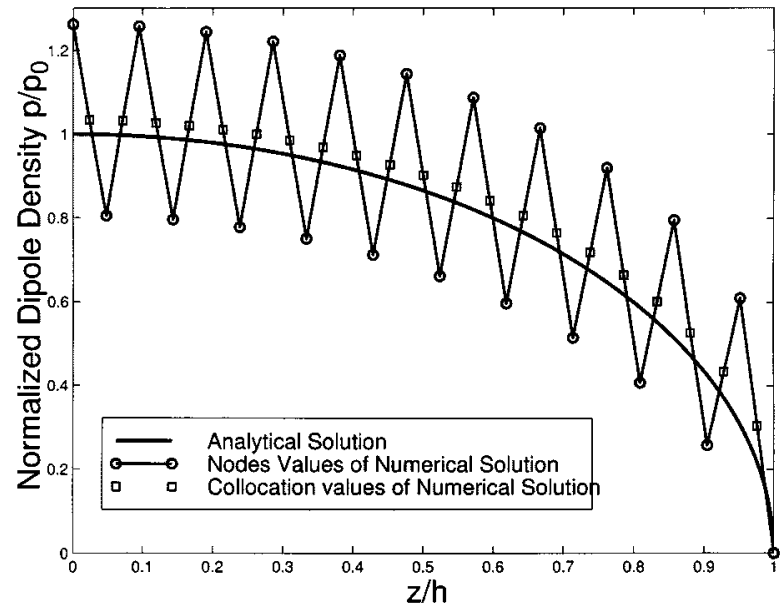

Fig. 4. Analytical and numerical solutions of the dipole density on a crack for a 2-D problem.

(e) is the eth element and $j$ is the local number of the node. The relation between both numbering is $n=\operatorname{num}(e, \mathrm{j})$. On each element, the dipole density is interpolated by shape functions $N_{j}^{(e)}: p^{(e)}(r)=\sum_{\text {nodes } j} p\left(\mathbf{r}_{n}\right) N^{(e, j)}(\mathbf{r})$. So, the linear system $\mathbf{J}=\mathbf{A p}$ is obtained. The elements of the vectors are $J_{m}=J_{0 n}\left(\mathbf{r}_{m}\right), p_{n}=p\left(\mathbf{r}_{n}\right)$. The $\mathbf{A}$ matrix elements are given by the sum

$$
A_{m n}=-k^{2} \sum_{e, j / n=\text { num }(e, j)} \iint_{S^{e}} G\left(\mathbf{r}_{m}, \mathbf{r}\right) N^{(e, j)}(\mathbf{r}) d S_{r} .
$$

If $\mathbf{r}_{m}$ locates in $S^{e}$ (self-term), the finite part of Hadamard to compute integral is used, else a Gaussian quadrature scheme is applied. The matrix is full and self-term is preponderant on the diagonal. Most elements far from the diagonal can be neglected, particularly reflection terms. A term $A_{m n}$ is neglected if

$$
A_{n m}<\left(10^{-4} A_{s}\right) / N_{n}
$$

where $A_{s}$ is the self-term and $N_{n}$ is the number of nodes. This criterion allows to decrease up to $70 \% \mathrm{CPU}$ time of matrix construct.

\section{A. Zeroth-Order Scheme}

There are piecewise constant functions. The collocation point and the node are at the middle of the rectangle. This method was already studied in [2] and [7]. The regularized formula proposed in [7] is a particular case of (7).

\section{B. First-Order Scheme}

The shape functions are bilinear. The mesh elements have four nodes at the corners. Because the gradient is not continuous at the nodes, the collocation point is at the middle of the element. (nonconforming element). Large oscillations of density $p$ arise between two elements. To illustrate this, let us consider a 2-D case of an infinite crack along $y$ and the low frequency limit case (direct current). The dipole density on the crack is given analytically [8]. Fig. 4 shows the numerical solution. Even if values at collocation points are correct, the computation fails at the nodes. In the 3-D case, similar oscillations arise.

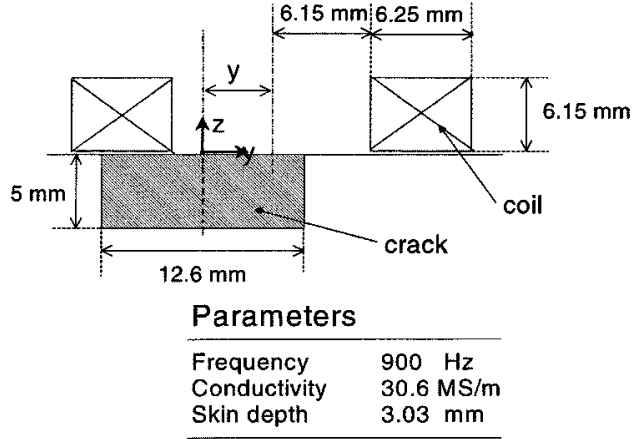

Fig. 5. The geometry and parameters of the studied problem.

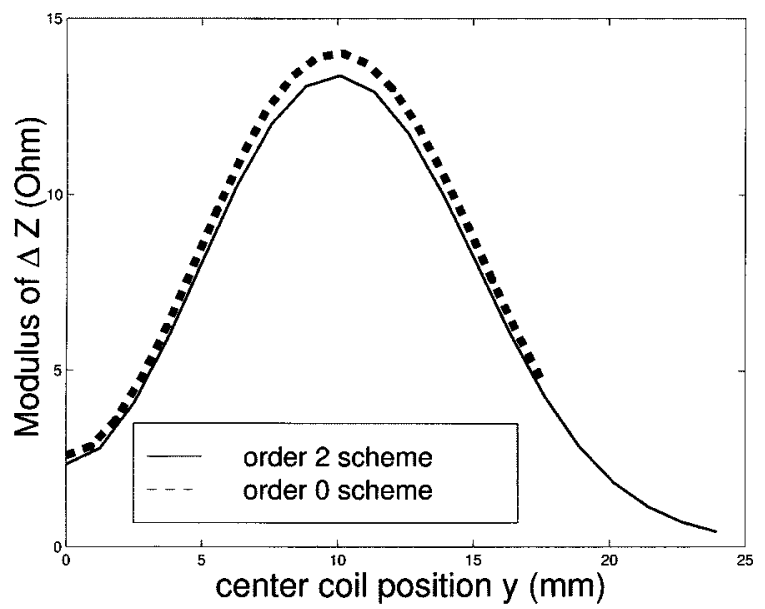

Fig. 6. TEAM Workshop Problem No. 15 validation. The modulus of impedance change computation versus the $y$ coordinate of the coil axis.

This is a consequence of the lack of the jump term. In fact, the self-term of first-order scheme is very close to the constant functions one. Thus, the numerical scheme cannot correctly evaluate the derivate of solution on each element.

The first-order scheme is not better than zeroth-order.

\section{Second-Order Scheme}

We consider nine nodes elements. The collocation point is in the middle of the rectangle. At the other nodes, we add gradient continuity requirements: two equations for corners and one equation at the middle of the element edge. The system is overdetermined, hence, it is solved in the least squares sense.

\section{RESULTS}

As a numerical example, the TEAM Workshop Problem no 15 has been solved. The studied geometry is shown in Fig. 5 . The thickness of the crack is assumed to be 0 , and we consider the plate as a half-space conductor.

\section{A. Validation}

Fig. 6 shows impedance change $\Delta Z$ for different coil positions for zeroth-order and second-order schemes for $20 \times 10$ and $10 \times 5$ elements, respectively. The relative difference is inferior to $8 \%$.

\section{B. Effectiveness of the Methods}

The influence of the mesh size (nodes number) on the $\Delta Z$ computation accuracy (Fig. 7) is studied. The difference be- 


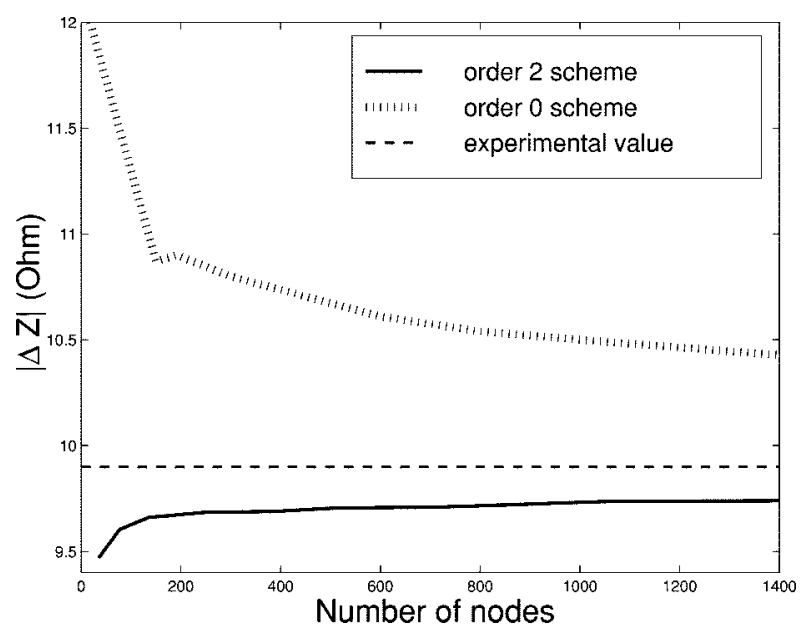

Fig. 7. Impedance change for the coil position $y=13 \mathrm{~mm}$ computed with zero and second-order schemes versus nodes number.

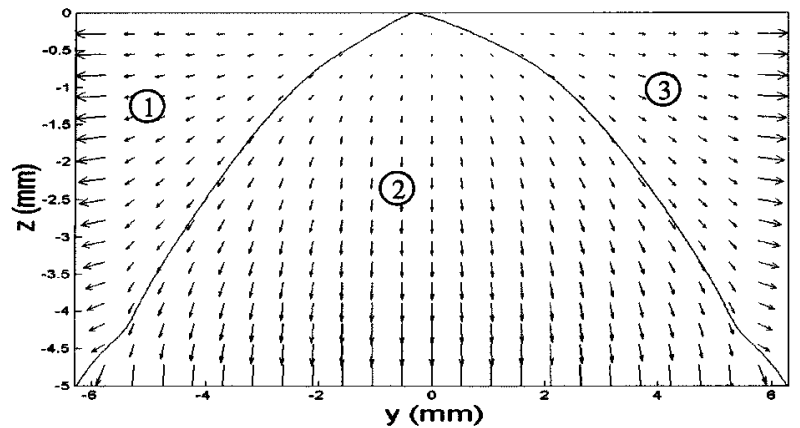

Fig. 8. Eddy-current distribution (arrows) on the crack. The solid lines are the demarcations between regions 1,2 , or 3 .

tween the both schemes is of order 5\%. The zeroth-order overestimates the impedance change. The main reason is that constant shape functions do not vanish at the crack edges. Bowler [2] has used a weight factor to minimize this effect. This problem does not occur for second-order scheme. Another source of numerical errors of zeroth-order scheme is that shape function derivates at the collocation point vanish. Nevertheless, for the FP integration (contrary to classical integration) the derivates have an important influence on the result: $50 \%$ of the self-term for a square element of length $\delta / 3$. This influence decreases with element size. For constant functions at each collocation point, this influence is neglected, thus the solution is accurate only for fine meshes.

The second-order scheme underestimates the experimental result. A reason is that we have neglected the crack width. A grid of $10 \times 5$ elements gives a good estimation: error inferior to $2 \%$.

\section{Example of Eddy-Current Distribution}

Electrical field is obtained by the gradient of $p$. This gradient is evaluated with formal derivation, so no numerical error is introduced. Fig. 8 shows eddy-current distribution at the coil position $y=9.5 \mathrm{~mm}$ (impedance change maximum). In region 1, eddy-current flows round left the crack: $14 \%$ of the incident current. In region 2, eddy-current flows under the crack (49\%). And in region 3, eddy-current flows round right the crack (37\%).

\section{CONCLUSION}

A simple physical interpretation of hypersingular integral is given. Two methods of regularization are described. These formulas allow to accurately evaluate the finite part of Hadamard for arbitrary shape functions, which have regularity requirements. A collocation method to solve integral equation is developed using constant, bilinear, and quadratic shape functions. An oscillation problem arises for linear scheme. The quadratic scheme is more effective than zeroth-order scheme.

\section{APPENDIX}

Krishnasamy et al. [4] shows the general relationship for hyper singular integral

$$
\begin{aligned}
I_{S} & =\lim _{\mathbf{r}^{\prime} \rightarrow \mathbf{r}_{0}} \iint_{S} \Delta_{T} g_{0}\left(R_{T}\right) p(\mathbf{r}) d S_{r} \\
& =F P \iint_{S} \Delta_{T} g_{0}(R) p(\mathbf{r}) d S_{r}+\nabla p \cdot \mathbf{C}
\end{aligned}
$$

where $\mathbf{C}=\left(c_{y}, c_{z}\right)$. The second term added to the $F P$ is the jump term. The constant $c_{y}$ is defined by the limit

$$
c_{y}=\lim _{d \rightarrow 0} \iint_{D} \Delta_{T} g_{0}(R) R \cos \theta d S_{r} .
$$

Let us show $c_{y}=0$. In order to simplify the proof, we suppose that the crack surface is plane. Integration by parts gives

$$
\begin{aligned}
c_{y}=-\lim _{d \rightarrow 0} \iint_{D} \frac{\partial g_{0}}{\partial R} & \cos \theta d S_{r} \\
& +\left.\lim _{d \rightarrow 0} \oint_{\Gamma} d \frac{\partial g_{0}}{\partial R}\right|_{R=d} \cos \theta d s .
\end{aligned}
$$

The first integral is interpreted as the rest of CPV, thus, it tends to zero with $d$. The second one, for symmetry reason, is zero. Then, $c_{y}=0$. In analogous way, $c_{z}=0$. In conclusion, the jump term is null.

\section{REFERENCES}

[1] J. L. Rasolonjanahary, F. Thollon, and N. Burais, "Study of eddy currents nondestructive testing system in riveted assemblies," IEEE Trans. Magn., vol. 32, pp. 1585-1588, May 1996.

[2] J. Bowler, "Eddy-current interaction with an ideal crack. The forward problem," J. Appl. Phys., vol. 75, no. 12, pp. 8128-8137, 1994.

[3] H. Igarashi and T. Honma, "An analysis of thin magnetic materials using hypersingular integral equations," IEEE Trans. Magn., vol. 32, pp. 682-685, May 1996.

[4] G. Krishnasamy, F. J. Rizzo, and T. J. Rudolphi, "Hypersingular boundary integral equations: Their occurrence, interpretation, regularization and computation," in Developments in Boundary Elements Methods, P. K. Banerjee and S. Kobayashi, Eds. Amsterdam, The Netherlands: Elsevier, 1994, vol. 7, ch. 6.

[5] E. Durand, Electrostatique I-Les Distributions. Paris, France: Masson, 1964, ch. 2, pp. 88-98

[6] M. Guiggiani, G. Krishnasamy, J. T. Rudolphi, and F. J. Rizzo, “A general algorithm for the numerical solution of hypersingular boundary integral equations," ASME J. Appl. Mech., vol. 59, pp. 604-614, 1992.

[7] C. Dezhi, K. R. Shao, S. Jianni, and Y. Weili, "Eddy current interaction with a thin-opening crack in a plate conductor," IEEE Trans. Magn., vol. 36, pp. 1745-1749, July 2000.

[8] D. Mishekar-Syahkal, R. Collins, and D. H. Michael, "The influence of skin depth on crack measurement by the ac field technique," J. Nondestructive Eval., vol. 3, no. 2, pp. 65-76, 1982. 